\title{
FIRST MEASUREMENT OF THE LIFETIME
}

OF THE CHARMED STRANGE BARYON $\Xi_{C}^{0}$

\author{
(ACCMOR Collaboration)

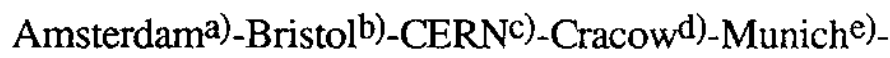 \\ Rutherfordf)_Valenciag) Collaboration
}

\begin{abstract}
S. Barlage1, H. Beckere2, T. Böhringerc 3 , M. Bosmane, V. Castilloc4, V. Chabaudc, C. Damerellf, C. Daum ${ }^{a}$, H. Dietle, A. Gillmanf, R. Gilmoreb ${ }^{b}$, T. Gooch ${ }^{b}$, L. Görlich ${ }^{d}$, P. Grasg, Z. Hajduk ${ }^{d}$, E. Higong, D.P. Kelseyc5, R. Klannere6, S. Kwanc, B. Lückingc, G. Lütjens ${ }^{\mathrm{e}}$, G. Lutz ${ }^{\mathrm{e}}$, J. Malos ${ }^{\mathrm{b}}$, W. Männere ${ }^{\mathrm{e}}$ E. Neugebauere7, H. Palka ${ }^{\mathrm{d}}$, M. Pepéf8, J. Richardson ${ }^{\mathrm{f} 9}$, K. Rybickid, H.J. Seebrunnerc, U. Stierline, H.G. Tieckea, G. Waltermanne, S. Watts ${ }^{\text {h }}$, P. Weilhammerc ${ }^{\text {e F. Wickens }}{ }^{\mathrm{f}}$, L.W. Wiggers ${ }^{a}$, M. Witek ${ }^{d}$ and T. Zeludziewicz ${ }^{\text {d10 }}$
\end{abstract}

\begin{abstract}
We have observed four unambiguous decays of the charmed strange baryon $\Xi_{c}^{0}$ in the NA32 experiment at CERN. Charge-coupled devices and silicon microstrip detectors were used to reconstruct the decay mode $\Xi_{\mathrm{C}}^{0} \rightarrow \mathrm{pK}-\overline{\mathrm{K}}^{*}(892)^{0}$ seen in events produced by the interaction of $230 \mathrm{GeV} / \mathrm{c}$ negative pions and kaons on a copper target. We present the first measurement of the lifetime of the $\Xi_{\mathcal{C}}^{0}$, together with a determination of its mass and production cross-section. The resonant components of the $\Xi_{c}^{0}$ decay are studied. We use our earlier measurement of the mass of the $\Xi_{\mathrm{c}}^{+}$in the determination of the isospin mass splitting of the $\Xi_{\mathrm{c}}$ states.
\end{abstract}

Submitted to Physics Letters B
a) NIKHEF-H, Amsterdam,NL
b) Univ. of Bristol, Bristol, UK
c) CERN, Geneva, CH
d) Inst. of Nucl. Physics, Cracow, Poland
c) Max-Planck-Inst. f. Physik, Munich, FRG
f) Rutherford Appleton Lab., Chilton, Didcot, UK
g) IFIC, CSIC \& Univ. of Valencia, Valencia, Spain
h) Brunel Univ., Uxbridge, Mddx, UK
1) Now at KNMI, De Bilt, NL
2) Now at Gesamthochschule, Saarbrücken, FRG
3) Now at University of Lausanne, Lausanne, $\mathrm{CH}$
4) Now at Univ. of Valencia, Valencia, Spain
5) Now at Rutherford Appleton Lab., Chilton, Didcot, UK
6) Now at DESY, Hamburg, FRG
7) Now at Universität-GH Siegen, Siegen, FRG
8) Now at CERN, Geneva, Switzerland
9) Now at Univ. of Geneva, Geneva, $\mathrm{CH}$
10) Now at University of Melbourne, Melbourne, Australia 


\section{INTRODUCTION}

For more than ten years now, many experiments have studied the properties of charmed mesons. The situation regarding charmed baryons is much less clear and it is only recently that experiments have been able to make progress in this difficult subject. Four charmed baryons are expected to decay weakly : $\Lambda_{\mathrm{c}}^{+}$(cud), $\Xi_{\mathrm{c}}^{+}(\mathrm{csu}), \Xi_{\mathrm{c}}^{0}(\mathrm{csd})$ and $\Omega_{\mathrm{c}}^{0}(\mathrm{css})$. Only the first two appear in the Stable Particle Table of the latest Review of Particle Properties [1]. The $\Lambda_{\mathrm{C}}^{+}$is well established; we have recently published a measurement of its mass and lifetime [2]. The $\Xi_{c}^{+}$was first observed by the hyperon beam WA62 experiment at CERN [3] and subsequently confirmed by the E400 experiment at FNAL [4].

More recently, we have reported the observation of two new decay modes of the $\Xi_{\mathrm{C}}^{+}$ $\left.\left(\Xi^{-} \pi^{+} \pi^{+} \text {and } \Sigma^{+} K^{-} \pi^{+}\right)^{*}\right)$ and measured its mass and lifetime to be $2466.5 \pm 2.7 \pm 1.2 \mathrm{MeV} / \mathrm{c}^{2}$ and $(2.0 \pm 1.1) \cdot 10^{-13}$ s respectively [5]. The CLEO Collaboration has published a year ago the first observation of $\Xi_{c}^{0}$ (decaying to $\Xi^{-} \pi^{+}$) [6] and also confirmed the $\Xi_{c}^{+} \rightarrow \Xi^{-} \pi^{+} \pi^{+}$decay mode [7]. They have measured the masses of the $\Xi_{c}^{+}$and $\Xi_{c}^{0}$ to be $2467 \pm 3 \pm 5 \mathrm{MeV} / \mathrm{c}^{2}$ and $2472 \pm 3 \pm 4 \mathrm{MeV} / \mathrm{c}^{2}$ respectively and the isospin mass splitting $\mathrm{M}\left(\Xi_{\mathrm{c}}^{+}\right)-\mathrm{M}\left(\Xi_{\mathrm{c}}^{0}\right)$ to be $-5 \pm 4 \pm 1$ $\mathrm{MeV} / \mathrm{c}^{2}$. In this letter we present data confirming the existence of the $\Xi_{c}^{0}$, seen in a new decay mode $\mathrm{pK}^{-} \overline{\mathrm{K}}^{*}(892)^{0}$, and measure its lifetime for the first time.

The data was taken at the CERN SPS in 1985-86 and was a continuation of the 1984 NA32 experiment [8]. The main aim of this new phase was to study the hadroproduction and decay of charmed particles into hadronic final states containing a $\mathrm{pK}^{-}$or $\mathrm{K}^{+} \mathrm{K}^{-}$pair, particularly $\Lambda_{C}^{+}$and $D_{S}^{+}$. We used the ACCMOR spectrometer [9] with an improved vertex detector containing charge-coupled devices, CCDs [10], for the first time. A modified version of the FAMP trigger [11] was used to record a total of 17 million events containing a pair of opposite charge kaons and/or protons.

\section{EXPERIMENTAL SETUP}

The NA32 experiment was situated in the North Area of the CERN SPS and used an unseparated negative beam with a momentum of $230 \mathrm{GeV} / \mathrm{c}$. Two CEDAR Cerenkov counters tagged incident pions $(96 \%)$ and kaons $(4 \%)$. The vertex detector consisted of a beam telescope of 7 silicon microstrip detectors (MSDs) and a vertex telescope of 2 CCDs and 8 MSDs. The CCDs, situated $1 \mathrm{~cm}$ and $2 \mathrm{~cm}$ in a vacuum downstream of a $2.5 \mathrm{~mm}$ thick Copper target, measured high precision space points $(\sim 5 \mu \mathrm{m})$ on tracks close to the production vertex. Straight line tracks are reconstructed with a precision on their transverse position of approximately $\left[5^{2}+(18 / \mathrm{p})^{2}\right]^{\frac{1}{2}} \mu \mathrm{m}$, where the momentum dependent term ( $\mathrm{p}$ is in GeV/c) is due to

*) Unless explicitly stated, a particle symbol stands for particle and antiparticle. 
multiple scattering. The high precision vertex detector and the lack of material for producing secondary interactions allow the clean reconstruction of charm decays with very few background events. The choice of a thin target $(2.5 \mathrm{~mm})$ was essential to enable the observation of charmed particles with very short lifetimes.

The large acceptance ACCMOR spectrometer consisted of two magnets and 48 planes of drift chambers [9]. Three multicellular threshold Cerenkov counters served to identify $\pi, K, p$ in the momentum range 4 to $80 \mathrm{GeV} / \mathrm{c}$. A two-level trigger was used to increase by a factor of 5 the sensitivity of the experiment to decays containing oppositely charged pairs of kaons and/or protons. The decay mode presented in this letter, $\Xi_{\mathrm{C}}^{0} \rightarrow \mathrm{pK}^{-} \overline{\mathrm{K}}^{* 0}$, more than satisfies our trigger conditions. More details on the trigger are given in Ref. 9.

\section{DATA ANALYSIS}

The method used to search for decays is very similar to that applied in our $\Lambda_{c}^{+}$analysis [2]. Briefly the data analysis consists of the following steps (more details may be found in Reference 2).

Firstly, all tracks are reconstructed separately in the beam and vertex telescopes and the drift chambers of the spectrometer and then the vertex telescope and drift chamber tracks are matched together. Particles are identified using the information from the Cerenkov detectors.

The next step is to search for vertices. The primary vertex is reconstructed from the beam track and as many of the outgoing tracks as possible. For those events where the primary vertex is found to be inside the copper target and where there are at least two tracks which do not originate from the primary vertex, a search is made for secondary vertices. Events are selected containing at least one secondary vertex outside of the copper (to remove secondary interactions). Strange particle decays $\left(\mathrm{K}^{0}, \Lambda^{0}\right)$ are removed. Approximately 300,000 events remain at this stage.

The secondary vertices are then scanned for fully reconstructed charm decays with no missing neutral particles, by restricting to those decays where the momentum vector sum of the decay products points back to the primary vertex $\left(\chi^{2}\right.$ probability greater than $\left.1 \%\right)$. Invariant mass distributions consistent with the particle identification are then plotted. In our $\Lambda_{c}^{+}$analysis [2], for example, the mass distribution for the $\mathrm{pK}^{-} \pi^{+}$channel contained a clear $\Lambda_{c}^{+}$signal of 135 events above a background of 31 . The background was mainly due to reflections from other charm particle decays where there was an ambiguity either in the topology of the decay or in the particle identification. The precise measurement of space points by our vertex detector allows the clean reconstruction of charm decays in a purely topological way with no restriction 
to particular decay modes. Using this method we have observed rare decay modes which have not been previously seen [12].

The invariant mass distribution for the channel $\mathrm{pK}^{-} \mathrm{K}^{-} \pi^{+}$is shown in Fig. 1a. We have removed those events with secondary vertices which are consistent with interactions in the CCDs and we have also demanded that all tracks have hits in at least one CCD. There are 33 decays passing the above cuts in the mass range $2.0-3.0 \mathrm{GeV} / \mathrm{c}^{2}$. The bin with the largest content is that centred at $2475 \mathrm{MeV} / \mathrm{c}^{2}$, suggesting that this may be evidence for a $\Xi_{\mathrm{C}}^{0}$ signal, although at this stage there is a substantial background.

In the case of our $\Lambda_{c}^{+}$analysis, further cuts were applied to the data, improving the ratio of signal to background by almost a factor 4 . One of these was to demand that all three tracks were displaced from the primary vertex $(3 \sigma, 3 \sigma$ and $1 \sigma)$. As it is possible that the $\Xi_{\mathrm{c}}^{0}$ has a very short lifetime, we were unable to use this approach here as this would have reduced our acceptance too much. However, other cuts could be used to purify the $\mathrm{pK}^{-} \mathrm{K}^{-} \pi^{+}$sample, as follows :

i) Reflections from other fully reconstructed charm events are removed (see Fig. 1b). The 7 decays which are cut are mainly due to $\Lambda_{c}^{+} \rightarrow \mathrm{pK}^{-} \pi^{+}$or $\mathrm{D}^{0} \rightarrow \mathrm{K}^{+} \mathrm{K}^{-} \pi^{+} \pi^{-}$where there is an additional pointing track superimposed in the first case and where the particle identification is ambiguous in both cases.

ii) By studying the $\Lambda_{c}^{+} \rightarrow \mathrm{pK}^{-} \pi^{+}$decays we define a tighter particle identification algorithm for selecting "non-pions" (i.e. kaons and protons). Demanding two such non-pions per decay in the $\Lambda_{c}^{+}$sample only reduces the number of accepted decays by $15 \%$, but at the same time decreases the background by a factor of two. Many of the events in Fig. 1b contain particles which could be either kaons or pions according to our looser identification cuts. We therefore demand three non-pions in the $\mathrm{pK}^{-} \mathrm{K}^{-} \pi^{+}$events thereby reducing the sample to 12 events (see Fig. 1c).

iii) In our $\Xi_{\mathrm{c}}^{+}$analysis [5] we found that the three decays to $\Sigma^{+} \mathrm{K}^{-} \pi^{+}$were all consistent with the $\mathrm{K}^{-} \pi^{+}$originating from a $\overline{\mathrm{K}}^{*}(892)^{0}$. Following this, we demand that one of the two $\mathrm{K}^{-} \pi^{+}$combinations have an effective mass within the range 796 to $996 \mathrm{MeV} / \mathrm{c}^{2}$ (i.e. $\pm 2 \Gamma$ ). Figure $1 \mathrm{~d}$ shows the resulting distribution : four events cluster about a mass of approximately $2470 \mathrm{MeV} / \mathrm{c} 2$ and there are only another three events, all above a mass of $2900 \mathrm{MeV} / \mathrm{c}^{2}$.

Given the very clean signal with virtually no background, these four events are clear evidence for the decay mode $\Xi_{\mathrm{c}}^{0} \rightarrow \mathrm{pK}^{-} \overline{\mathrm{K}}^{*}(892)^{0}$. Three of the decays are $\Xi_{\mathrm{c}}^{0}$ while the fourth one is a $\bar{\Xi}_{c}^{0}$. Two of the $\Xi_{c}^{0}$ and the $\bar{\Xi}_{c}^{0}$ are produced by incoming pions while the re-maining $\Xi_{c}^{0}$ is produced by an incoming $\mathrm{K}^{-}$. In the latter case the $\Xi_{\mathrm{c}}^{0}$ contains an s-quark, like the beam particle. More details of the decays are presented in Table 1. 


\section{RESULTS}

The results presented here are based on the full data sample of 17 million triggers. The weighted mean mass of the four $\Xi_{\mathrm{c}}^{0} \rightarrow \mathrm{pK}-\overline{\mathrm{K}}^{* 0}$ decays is $2473.3 \pm 1.9 \mathrm{MeV} / \mathrm{c}^{2}$ in good agreement with the CLEO measurement of $2472 \pm 3 \pm 4 \mathrm{MeV} / \mathrm{c}^{2}[5,6]$. As in the case of our $\Lambda_{\mathrm{c}}^{+}$analysis [2], we estimate the systematic uncertainty on the $\Xi_{\mathrm{c}}^{0}$ mass to be $\pm 1.2 \mathrm{MeV} / \mathrm{c}^{2}$. Combining this with the $\Xi_{c}^{+}$mass of $2466.5 \pm 2.7 \mathrm{MeV} / \mathrm{c}^{2}$ from our earlier letter [5], we measure an isospin mass splitting of $\mathrm{M}\left(\Xi_{\mathrm{c}}^{+}\right)-\mathrm{M}\left(\Xi_{\mathrm{c}}^{0}\right)=-6.8 \pm 3.3 \mathrm{MeV} / \mathrm{c}^{2}$. The systematic error in this case is much smaller because of several effects which cancel out; we estimate it to be approximately $\pm 0.5 \mathrm{MeV} / \mathrm{c}^{2}$. This result, taken together with that of CLEO, indicates that the $\Xi_{c}^{0}$ is heavier than the $\Xi_{c}^{+}$, which puts a restriction on several theoretical predictions [see Reference 13 for an extensive discussion].

The mean uncorrected flighttime of the four $\Xi_{\mathrm{c}}^{0}$ decays is $2.4 \cdot 10^{-13} \mathrm{~s}$. To measure the $\Xi_{\mathrm{c}}^{0}$ lifetime, we must correct the observed decay lengths for the acceptance of the cuts of our analysis. For each event, we determine the minimum detectable decay length $\boldsymbol{\ell}_{\min }$ and calculate the corrected proper time $\mathrm{t}=\left(\boldsymbol{\ell}-\boldsymbol{\ell}_{\min }\right) \mathrm{M}\left(\Xi_{\mathrm{c}}\right) / \mathrm{cP}\left(\Xi_{\mathrm{c}}\right)$, where $\boldsymbol{\ell}$ and $\mathrm{P}\left(\Xi_{\mathrm{c}}\right)$ are the decay length and momentum of the $\Xi_{c}^{0}$ decay. The resulting mean $\Xi_{c}^{0}$ lifetime is $\left(0.82 \pm_{0.30}\right) \cdot 10^{-13} \mathrm{~s}$. We have checked for possible sources of systematic error on the $\Xi_{c}^{0}$ lifetime. These include a non-constant geometrical acceptance as a function of decay position, distortion in the reconstruction of secondary vertices and a varying efficiency for finding vertices within the decay volume. In a similar way to our $\Lambda_{c}^{+}$analysis [2], we find that the systematic error is negligible compared to the quoted statistical errors. This is the first measurement of the $\Xi_{\mathrm{C}}^{0}$ lifetime and is the shortest charmed particle lifetime ever measured.

Two theoretical calculations of charmed baryon lifetimes have been published [14,15]. These are based on a spectator diagram, $W$-exchange and quark interference with QCD effects. Guberina et al. [14] predict $\tau\left(\Omega_{\mathrm{c}}^{0}\right) \approx \tau\left(\Xi_{\mathrm{c}}^{0}\right)<\tau\left(\Lambda_{\mathrm{c}}^{+}\right)<\tau\left(\Xi_{\mathrm{c}}^{+}\right)$, whereas Voloshin and Shifman [15] predict $\tau\left(\Omega_{\mathrm{c}}^{0}\right)<\tau\left(\Xi_{\mathrm{c}}^{0}\right)<\tau\left(\Lambda_{\mathrm{c}}^{+}\right) \approx \tau\left(\Xi_{\mathrm{c}}^{+}\right)$, where each inequality represents a factor between 1.5 and 2.0. Our measurements of $\tau\left(\Xi_{\mathrm{c}}^{0}\right), \tau\left(\Xi_{\mathrm{c}}^{+}\right)=\left(2.0 \pm_{0.6}^{1.1} \cdot 10^{-13_{\mathrm{S}}}[5]\right.$ and $\tau\left(\Lambda_{\mathrm{c}}^{+}\right)=$ $(1.96 \pm 0.23) \cdot 10^{-13} \mathrm{~S}[2]$ support the latter prediction, although more statistics is required to make a firm conclusion.

We have searched for resonant components in the $\Xi_{c}^{0}$ decays. There are two $\mathrm{K}^{-} \pi^{+}$combinations per decay. The presence of at least one $\overline{\mathrm{K}}^{*}(892)^{0}$ was demanded in the extraction of the signal, but as may be seen in Table 1 , events 2 and 4 have two $\mathrm{K}^{-} \pi^{+}$masses within $\pm 2 \Gamma$ of the $\overline{\mathrm{K}}^{*}$ mass. There are also two $\mathrm{pK}^{-}$mass combinations per decay. For events 1 and 3, using the kaon which is not part of the $\overline{\mathrm{K}}^{*}$ combination, the $\mathrm{pK}^{-}$masses are found to be 1511 and $1525 \mathrm{MeV} / \mathrm{c}^{2}$ respectively. For event 4 , the $\mathrm{pK}^{-}$masses corresponding to the $\overline{\mathrm{K}}^{*}$ combinations are 1529 and $1545 \mathrm{MeV} / \mathrm{c}^{2}$. These $\mathrm{pK}^{-}$masses are compatible with the decay of the narrow 
$\Lambda(1520)$ resonance, suggesting that the $\Xi_{\mathcal{C}}^{0}$ might decay to $\Lambda(1520) \overline{\mathrm{K}}^{*}(892)^{0}$. We cannot interpret these data as being conclusive evidence for $\Lambda(1520)$ because the available phase space tends to force the $\mathrm{pK}^{-}$mass into the region of the $\Lambda(1520)$.

Before we can measure the total production cross-section of $\Xi_{c}^{0}$ by pions, we must determine the acceptance of the geometry of our experimental setup, the trigger and the cuts of the analysis. This is calculated in a Monte Carlo simulation of $\Xi_{c}^{0}$ production using the usual distribution $\mathrm{d}^{2} \sigma / d \mathrm{x}_{\mathrm{F}} d \mathrm{p}_{\mathrm{t}}^{2} \sim\left(1-\mathrm{x}_{\mathrm{F}}\right)^{\mathrm{n}} \mathrm{e}^{-b \mathrm{p}_{\mathrm{t}}^{2}}$ where $\mathrm{x}_{\mathrm{F}}$ and $\mathrm{p}_{\mathrm{t}}$ are the Feynman variable and transverse momentum of the $\Xi_{C}^{0}$. We assume a similar production mechanism to that we have observed for $\Lambda_{c}^{+}$[16], i.e. $n=3$ and $b=1 \mathrm{GeV}^{-2}$, and use the $\Xi_{\mathrm{c}}^{0}$ lifetime as presented in this letter. The $\Xi_{c}^{0}$ are simulated together with $\mathrm{D}$ mesons $\left(67 \% \overline{\mathrm{D}}^{0}\right.$ and $\left.33 \% \mathrm{D}^{-}\right)$and merged with additional tracks from real events. The acceptance for the channel $\Xi_{\mathrm{c}}^{0} \rightarrow \mathrm{pK}-\overline{\mathrm{K}} * 0$ is found to be $1.1 \%$, including allowance for the non-observed all neutral decay mode of the $\overline{\mathrm{K}}^{*}(892)^{0}$.

Assuming a linear A-dependence, we measure from the three pion induced $\Xi_{c}^{0}$ events a $\Xi_{c}^{0}$ production cross-section times branching fraction of $\Xi_{\mathrm{c}}^{0} \rightarrow \mathrm{pK}^{-} \overline{\mathrm{K}}^{*}(892)^{0}$ to be $0.019 \pm 0.011 \pm 0.066 \mu \mathrm{b}$ per nucleon for $\mathrm{x}_{\mathrm{F}}>0$. The first error is statistical, the second one systematic and reflects mainly the uncertainties on the $\Xi_{c}^{0}$ lifetime, on the value of $n$ and on the trigger simulation (for $n$ we assume an uncertainty of \pm 2 ).

We have looked for the decay mode $\Xi_{c}^{0} \rightarrow \Xi^{-} \pi^{+}$as observed by CLEO [6]. $\Xi^{-}$particles are reconstructed via their decay into $\Lambda^{0} \pi^{-}$as in our $\Xi_{c}^{+}$analysis [5]. Selecting two-prong secondary vertices containing a $\Xi^{-}$and $\pi^{+}$with both tracks displaced from the primary vertex results in 8 decays all of which have effective masses less than $2110 \mathrm{MeV} / \mathrm{c}^{2}$. The decay of $\Xi_{c}^{0}$ to $\Xi^{-} \pi^{+}$would be highly asymmetric in the laboratory frame making the $\Xi^{-}$travel forwards; relaxing the requirement that the $\Xi^{-}$be displaced from the primary vertex increases the number of selected events to 160 but all masses are less than $2170 \mathrm{MeV} / \mathrm{c}^{2}$. We therefore do not observe $\Xi_{c}^{0} \rightarrow \Xi^{-} \pi^{+}$.

We have reported here on the possible observation of the decay mode $\Xi_{c}^{0} \rightarrow$ $\Lambda(1520) \overline{\mathrm{K}} * 0$. In addition to decaying to $N \mathrm{~K}$ the $\Lambda(1520)$ also decays to $\Sigma \pi$. We have therefore searched for the decay mode $\Xi_{\mathrm{c}}^{0} \rightarrow \Sigma^{+} \pi^{-} \mathrm{K}^{-} \pi^{+}$. Using very similar techniques to our analysis of $\Xi_{\mathrm{c}}^{+} \rightarrow \Sigma^{+} \mathrm{K}^{-} \pi^{+}$[5] we found no events in this channel. Given the fact that we are only able to reconstruct $\Sigma^{+}$, and not $\Sigma^{0}$ or $\Sigma^{-}$, and then only via its decay to $\mathrm{p} \pi^{0}$ means that the three possible $\Xi_{c}^{0}$ decays to $\Lambda(1520) \overline{\mathrm{K}}^{*}$ lead us to expect to see approximately one decay via $\Sigma+\pi^{-} \mathrm{K}^{-} \pi^{+}$, fully consistent with our null result in this channel. 


\section{CONCLUSIONS}

We have observed four unambiguous decays of $\Xi_{\mathrm{c}}^{0} \rightarrow \mathrm{pK}^{-} \overline{\mathrm{K}}^{*}(892)^{0}$. We measure the mass of $\Xi_{\mathrm{c}}^{0}$ to be $2473.3 \pm 1.9 \pm 1.2 \mathrm{MeV} / \mathrm{c}^{2}$ and the isospin mass splitting $\mathrm{M}\left(\Xi_{\mathrm{c}}^{+}\right)-\mathrm{M}\left(\Xi_{\mathrm{c}}^{0}\right)$ to be $-6.8 \pm 3.3 \pm 0.5 \mathrm{MeV} / \mathrm{c}^{2}$. We have measured the $\Xi_{\mathrm{c}}^{0}$ lifetime for the first time and find it to be $\left(0.82 \pm 0.59 \cdot 10^{-13} \mathrm{~s}\right.$. Assuming a similar production mechanism of $\Xi_{\mathrm{C}}^{0}$ by pions as for $\Lambda_{\mathrm{C}}^{+}$, we determine the $\Xi_{\mathrm{c}}^{0}$ production cross-section times branching fraction of $\Xi_{\mathrm{c}}^{0} \rightarrow \mathrm{pK}-\overline{\mathrm{K}}^{*}(892)^{0}$ to be $0.019 \pm 0.011 \pm_{0.009}^{0.066} \mu \mathrm{b}$ for $\mathrm{x}_{\mathrm{F}}>0$. Three of the decays are consistent with the $\mathrm{pK}^{-}$being decay products of the $\Lambda(1520)$ resonance.

\section{Acknowledgements}

We would like to thank R.L. English and A.L. Lintern for their work on the CCD detectors and readout electronics, A. Bjorkebo and $\mathrm{H}$. Karstens for processing large amounts of data and C. Ponting for her skilful typing of the manuscript. 


\section{REFERENCES}

[1] Review of Particle Properties, Phys. Lett. 204B (1988).

[2] S. Barlag et al., Phys. Lett. 218B (1989) 374.

[3] S.F. Biagi et al., Phys. Lett. 122B (1983) 455.

S.F. Biagi et al., Z. Phys. C28 (1985) 175.

[4] P. Coteus et al., Phys. Rev. Lett. 59 (1987) 1530.

[5] Proc. XII International Workshop on Weak Interactions and Neutrinos (Ginosar, Israel, April 1989); to be published in Nucl. Phys. B (Proc.Suppl.).

S. Barlag et al., CERN-EP/89-145; to be published in Physics Letters B.

[6] P. Avery et al., Phys. Rev. Lett. $\underline{62}$ (1989) 863.

[7] M.S. Alam et al., Phys. Lett. 226B (1989) 401.

[8] H. Becker et al., Phys. Lett. 184B (1987) 277.

S. Barlag et al., Z. Phys. C39 (1988) 451.

[9] S. Barlag et al., Phys. Lett. 184B (1987) 283.

[10] R. Bailey et al., Nucl. Instrum. Methods $\underline{213}$ (1983) 201.

[11] C. Daum et al., Nucl. Instrum. Methods 217 (1983) 361.

[12] S. Barlag et al. CERN-EP/88-103.

S. Barlag et al., Measurement of the Branching Ratios and Branching Fractions of the Charmed Meson $\mathrm{D}^{0}$; to be published in Physics Letters B.

[13] S.R. Klein, BU-HEP-89-16, Boston University, August 1989 and references therein.

[14] B. Guberina et al., Z. Phys. C33 (1986) 297.

[15] M.B. Voloshin and M.A. Shifman, Zh. Eksp. Teor. Fiz. 91 (1986) 1180.

[16] P. Gras, Ph.D. Thesis, University of Valencia, 1988.

S. Barlag et al., Production of the Charmed Baryon $\Lambda_{\mathrm{c}}^{+}$in $\pi^{-}-\mathrm{Cu}$ Interactions at $230 \mathrm{GeV} / \mathrm{c}$; to be submitted to Physics Letters B. 
Table 1

Details of the four $\Xi_{\mathrm{c}}^{0} \rightarrow \mathrm{pK}^{-} \mathrm{K}^{-} \pi^{+}$decays

\begin{tabular}{|c|c|c|c|c|c|c|c|c|}
\hline Event & \multicolumn{2}{|c|}{1} & \multicolumn{2}{|r|}{2} & \multicolumn{2}{|c|}{3} & \multicolumn{2}{|r|}{4} \\
\hline $\begin{array}{l}\text { Mass of }(\mathrm{pKK} \pi) \text { system } \\
\left(\mathrm{MeV} / \mathrm{c}^{2}\right)\end{array}$ & \multicolumn{2}{|c|}{$2470.5 \pm 3.7$} & \multicolumn{2}{|c|}{$2476.6 \pm 4.0$} & \multicolumn{2}{|c|}{$2478.1 \pm 4.0$} & \multicolumn{2}{|c|}{$2470.0 \pm 3.3$} \\
\hline Total momentum $(\mathrm{GeV} / \mathrm{c})$ & \multicolumn{2}{|r|}{123.1} & \multicolumn{2}{|r|}{56.6} & \multicolumn{2}{|r|}{129.2} & \multicolumn{2}{|r|}{65.8} \\
\hline $\begin{array}{l}\text { Distance of total momentum } \\
\text { vector to primary vertex } \\
\text { and its error }(\mu \mathrm{m})\end{array}$ & \multicolumn{2}{|c|}{$6.5(4.4)$} & \multicolumn{2}{|c|}{$3.6(3.8)$} & \multicolumn{2}{|c|}{$3.8(4.5)$} & \multicolumn{2}{|c|}{$1.9(3.5)$} \\
\hline $\begin{array}{l}\text { Distance of decay vertex to } \\
\text { target edge (standard devia- } \\
\text { tions) }\end{array}$ & & 5.2 & & 3.0 & & 19 & & 1.1 \\
\hline $\begin{array}{l}\text { Mass of }\left(\mathrm{K}^{-} \pi^{+}\right) \text {system } \\
2 \text { solutions }\left(\mathrm{MeV} / \mathrm{c}^{2}\right)\end{array}$ & 868 & 780 & 830 & 932 & 865 & 791 & 848 & 849 \\
\hline $\begin{array}{l}\text { Mass of associated } \\
\left(\mathrm{pK}^{-}\right) \text {systems }\left(\mathrm{MeV} / \mathrm{c}^{2}\right)\end{array}$ & 1511 & 1491 & 1561 & 1435 & 1525 & 1674 & 1529 & 1545 \\
\hline Decay length $\ell(\mathrm{mm})$ & & 2.47 & & 2.14 & & 4.42 & & 1.62 \\
\hline$\ell_{\min }(\mathrm{mm})$ & & 1.43 & & 1.68 & & 1.83 & & 1.41 \\
\hline Corrected lifetime $\left(10^{-13} \mathrm{~s}\right)$ & & 0.70 & & 0.67 & & 1.65 & & 0.26 \\
\hline Reaction & & $\rightarrow \Xi_{c}^{0}$ & & $\rightarrow \Xi_{\mathrm{c}}^{0}$ & & $\rightarrow \Xi_{\mathrm{C}}^{0}$ & & $\rightarrow \Xi_{\mathrm{C}}^{0}$ \\
\hline
\end{tabular}




\section{Figure Captions}

Fig. 1 Invariant mass distribution of the $\mathrm{pK}^{-} \mathrm{K}^{-} \pi^{+}$channel :
a) After standard cuts ( $\geq 2$ displaced tracks)
b) After removal of reflections.
c) After tightening particle identification.
d) After demanding $\overline{\mathrm{K}}^{* 0} \rightarrow \mathrm{K}^{-} \pi^{+}$. 

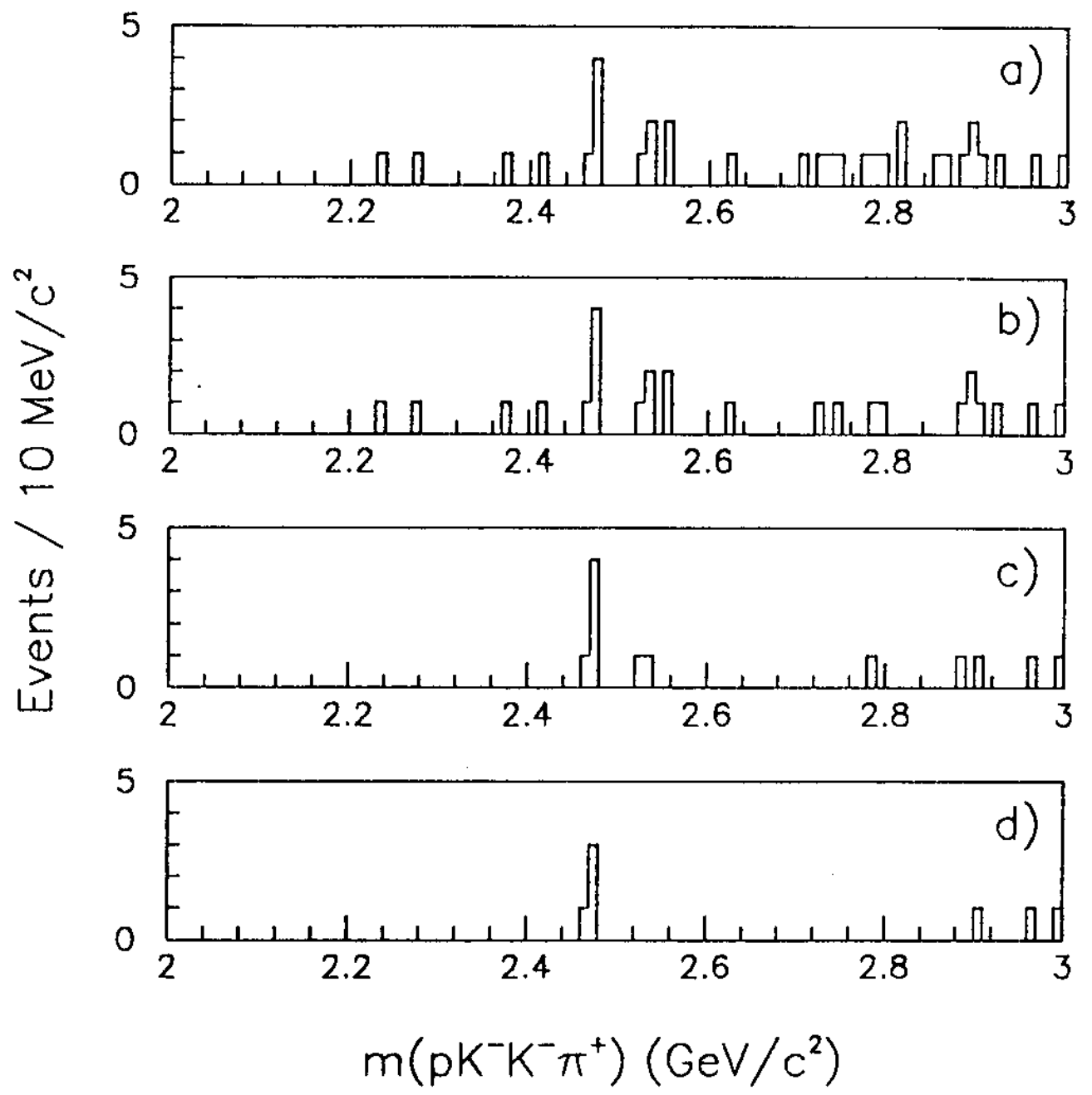

Fig. 1 